\title{
Die Popularklage zur Verteidigung der "Rechte Gottes": Hisba im heutigen Ägypten
}

\author{
Von Kilian Bälz
}

Wohl noch nie ist in der deutschen Presse über einen ägyptischen Prozeß so ausführlich berichtet worden wie über den "Fall Abû Zayd". Abû Zayd, Professor für arabische Literatur an der Kairo Universität, hatte mit seiner modernistischen Interpretation des Koran die erbitterte Kritik der ägyptischen Islamisten auf sich gezogen. Mit einer beim Familiengericht Giza im Jahr 1993 erhobenen Klage begehrte daraufhin eine Gruppe kairener Rechtsanwälte die Feststellung der Nichtigkeit seiner Ehe. Denn, so trugen die Kläger vor, die Ehe eines Apostaten mit einer Muslima sei nach islamischem Recht nichtig. Wer vom Glauben abgefallen sei, dürfe nicht mit einer Muslima verheiratet sein. Das Recht, in solch einem Fall die Unwirksamkeit der Ehe im Wege der Popularklage geltend zu machen, ergebe sich aus dem islamischen Prinzip der hisba. Denn die hisba gebiete einem jeden Muslim, "das Gute zu befördern und das Schlechte zu verhindern." Nachdem die Klage in der ersten Instanz als unzulässig abgewiesen worden war, gab das Appellationsgericht Kairo der Klage statt. Der ägyptische Kassationshof hat diese Entscheidung im August 1996 bestätigt. ${ }^{1}$

Der "Fall Abû Zayd" berührt eine ganze Reihe juristisch interessanter Fragen: ${ }^{2}$ Zum Beispiel, wie ein staatliches Gericht überhaupt darüber entscheiden kann, ob jemand vom Glauben abgefallen ist. Des weiteren die Frage der Eheauflösung aufgrund von Apostasie. Oder die Problematik der Rechtsstellung des Apostaten im allgemeinen und, damit in engem Zusammenhang stehend, das Problem von Meinungs-, Wissenschafts- und Religionsfreiheit.

In diesem Beitrag soll jedoch der Fall Abû Zayd zum Anlaß genommen werden, einer anderen Frage nachzugehen, und zwar dem islamischen Prinzip der hisba. Dieses Prinzip

1 Ägyptischer Kassationshof, Urt. v. 5.8.1996, E. 475, 478, 481 / 65. Vorinstanzen: Gericht der ersten Instanz Giza, Urt. v. 27.1.1994, E. 591/ 1993; Appellationsgericht Kairo, Urt. v. 14.6.1995, E. 287/ 111 .

2 Ausführlich zum "Fall Abû Zayd": Baudouin Dupret / Jean-Noël Ferrié, For intérieur et ordre public. Ou comment la problématique de l'Aufklärung peut permettre de décrire un débat égyptien, in: Gilles Boëtsch / Baudouin Dupret / Jean-Noël Ferrié (eds.), Droits et sociétes dans le monde arabe, Aix-en-Provence 1997, S. 193-215; Kilian Bälz, Submitting Faith to Judicial Scrutiny Through the Family Trial: The "Abû Zayd Case", Welt des Islams 37 (1997), S. 135-155. 
hat überhaupt erst die Grundlage dafür geschaffen, die Rechtgläubigkeit des ägyptischen Literaturwissenschaftlers im Wege einer Popularklage von einem staatlichen Gericht nachprüfen zu lassen. Das Institut der hisba ist dabei seit langem ein beliebter Gegenstand islamwissenschaftlicher und historischer Untersuchungen. ${ }^{3}$ Im folgenden sollen daher auch nicht dessen Ursprung oder seine Ausgestaltung im traditionellen islamischen Recht im Vordergrund stehen. Untersucht wird vielmehr der Funktionswandel der hisba, den diese in der heutigen ägyptischen Rechtsprechung erfahren hat.

I.

Was ist hisba? Der islamische Jurist al-Mâwardî (gest. 1058 n. Chr.), dessen Kitâb alahkâm al-sultâniyya (frei übersetzt: Handbuch des Staatsrechts) zu den anerkannten Klassikern der islamischen Staatsrechtslehre zählt, hat der hisba ein ganzes Kapitel gewidmet. Hisba, definiert al-Mâwardî, ist die Pflicht, "das Gute zu befördern, sollte bekannt werden, daß von ihm abgewichen wird und das Schlechte zu verhindern, sollte bekannt werden, daß es begangen wird." ${ }^{4}$ Diese Pflicht obliege zunächst einmal einem jeden Muslim: Jeder hat die Pflicht und das Recht zur hisba. Neben dieser "Jedermanns-hisba", so al-Mâwardî weiter, gibt es jedoch auch eine staatliche Institution, die mit der Aufgabe der hisba betraut ist. Dies ist der muhtasib, ${ }^{5}$ ein öffentliches Amt in der islamischen Stadt, das üblicherweise als "Marktaufseher" bezeichnet wird.

Dem muhtasib oblagen eine Vielzahl von Aufgaben, ${ }^{6}$ die nach heutigem Verständnis Gegenstand des Polizei- und Ordnungsrechts sind: Er kontrollierte die Gewichte auf dem sûq und die Zuverlässigkeit der Gewerbetreibenden; ihm oblag die Überwachung des Freitagsgebetes und die Aufrechterhaltung der öffentlichen Moral. Der muhtasib schritt ein bei betrügerischem Geschäftsgebaren, öffentlicher Trunkenheit sowie bei unmoralischen Zuständen in den öffentlichen Bädern. Die Vorschriften, über deren Einhaltung der muhtasib wachte, werden dabei von den Juristen den "Rechtsansprüchen Gottes" [huqûq Allah]

Z.B. Cahen / Talbi, Artikel "Hisba", in: The Encyclopaedia of Islam, 2. Aufl., Leiden / London, 1960 ff.; Yassine Essid, A Critique of the Origins of Islamic Economic Thought, Leiden / New York / Köln, 1995, S. 111-177; Benjamin R. Foster, Agoranomos and Muhtasib, Journal of the Economic and Social History of the Orient 13 (1970), S. 124-144; Emst Klingmiiller, Agoranomos und Muhtasib. Zum Funktionswandel eines Amtes in Islamischer Zeit, in: Festschrift für Erwin Seidel, Köln, 1975, S. 88-98; Ann K.S. Lambton, State and Government in Medieval Islam, Oxford / New York, 1981, S. 310-313; Emile Tyan, Histoire de l'organisation judicaire en pays d'Islam, 2. Aufl., Leiden, 1960, S. 617-650. Al-Mâwardî, Al-ahkâm al-sultâniyya, zit. n. Ausgabe Dâr al-Kutub al-'Ilmî, Beirut, o.D., S. 299.

5 A.a.O.

6 Ausführlich zu den Aufgaben des muhtasib: Essid (Anm. 3) S. 137-172; Tyan (Anm. 3), S. 631650. 
zugeordnet. $^{7}$ Darunter werden die Bestimmungen des islamischen Rechts verstanden, die im Interesse der Allgemeinheit bestehen und der Disposition des einzelnen von daher entzogen sind. ${ }^{8}$

Wie verhält sich nun das Recht des einzelnen, aufgrund der hisba einzuschreiten, zum Amt des muhtasib, der mit der Ausübung der hisba hoheitlich betraut ist? Das Verhältnis von Jedermanns-hisba und dem staatlichen Amt wird von al-Mâwardî nicht ausdrücklich behandelt. Daß diese Frage dennoch auch die Juristen seiner Zeit beschäftigt hat, zeigt der "Leitfaden für die gottgefällige Ausübung der hisba" des Ägypters Ibn al-Ukhuwwa (gest. 1329 n.Chr.). ${ }^{9}$ Bei diesem Buch handelt es sich - im Gegensatz zu der eher theoretisch ausgerichteten Monographie al-Mâwardîs - um eine Darstellung für die Praxis. Der Autor, selbst langjähriger muhtasib, wollte hier seine praktischen Erfahrungen an die Nachwelt weitergeben. Der frühere muhtasib stand dabei der Jedermanns-hisba zumindest kritisch gegenüber: Zwar seien alle Muslime zur hisba berufen, konzediert auch Ibn al-Ukhuwwa. Doch sollten sie nicht selbst einschreiten, sondern diese nur mit Worten ausüben, indem sie auf Verstöße aufmerksam machen und diese der Obrigkeit anzeigen. ${ }^{10}$

Dies läßt den Willen erkennen, die Jedermanns-hisba zugunsten des staatlichen Amts zurücktreten zu lassen. Die Aufrechterhaltung der öffentlichen Sicherheit und Ordnung ist eine originär staatliche Aufgabe. Vieles spricht daher dafür, daß die hisba im traditionellen islamischen Recht als eine öffentliche Aufgabe verstanden wurde, die einer staatlichen Institution, dem muhtasib, übertragen war. Die individuelle Pflicht eines jeden Muslim zur Ausübung der hisba hat zwar in der Theorie fortbestanden, praktisch spielte sie aber keine Rolle.

II.

Ein genau entgegengesetztes Bild bietet die Rolle der hisba in der ägyptischen Rechtsprechung der letzten Jahre: Hier steht - das hat der eingangs erwähnte Fall Abû Zayd gezeigt -

Daneben konnte der muhtasib in bestimmten Fällen jedoch auch zum "Schutz privater Rechte" tätig werden, so zum Beispiel als eine Art Mahngericht; vgl. Tyan (Anm. 3), S. 632, 645-648.

8 Hierzu ausführlich: Baber Johansen, Eigentum, Familie und Obrigkeit im hanafitischen Strafrecht. Das Verhältnis der privaten Rechte zu den Forderungen der Allgemeinheit in hanafitischen Rechtskommentaren, Welt des Islams 19 (1979), S. 1-73.

Ibn al-Ukhuwwa, Ma'âlim al-qurba fî ahkâm al-hisba, zit. n. d. Ausgabe von Reuben Levy, Cambridge, 1938.

A.a.O. S. 22. 
die Jedermanns-hisba im Vordergrund. Denn das Amt des muhtasib ist bereits im Zuge der Verwaltungsreformen in der ersten Hälfte des 19. Jahrhunderts abgeschafft worden. ${ }^{11}$

Die Abschaffung des muhtasibs war dabei nur ein Aspekt der umfassenden Reformen von Recht und Verwaltung im Ägypten des 19. Jahrhunderts; ${ }^{12}$ mit Ausnahme des Familienrechts sind heute Kodifikationen nach europäischem Vorbild an die Stelle des islamischen Rechts getreten. Dies gilt auch für das Verwaltungsrecht. Die Ausführungsverordnung des "Gesetzes über die Lokalverwaltung" (Verordnung des Ministerpräsidenten 707/ 1979) bestimmt dabei in Art. 27: "Der Gouverneur [muhâfiz] ist für die Aufrechterhaltung der Sicherheit und der öffentlichen Moral zuständig."

In Anbetracht der in dieser Vorschrift zum Ausdruck kommenden Kompetenzverteilung stellt sich die Frage, ob es daneben noch ein Recht des einzelnen geben kann, das "Gute zu befördern und das Schlechte zu verhindern," das auf das islamische Recht gestützt wird. Dies hat die ägyptische Rechtsprechung wiederholt beschäftigt, und zwar schon lange, bevor die hisba durch den Fall Abû Zayd in das Blickfeld der Öffentlichkeit geriet. Die Grundsatzentscheidung des ägyptischen Kassationshofes zu dieser Frage stammt aus dem Jahr $1966 .{ }^{13}$ Hier ging es um folgenden Sachverhalt:

Eine Muslima hatte den christlichen Glauben angenommen, um einen Kopten kirchlich heiraten zu können. Nachfolgend lebten die Eheleute in einer Mietwohnung in Mansura. Der Vermieter wollte die Wohnung anderweitig vermieten und deshalb den Eheleuten kündigen. Mit diesem Begehren war er in allen Instanzen an dem sehr mieterfreundlichen ägyptischen Wohnraummietrecht gescheitert. Da erhob er eine Klage vor dem Familiengericht, mit der er begehrte festzustellen, daß die Ehe der Mieter nichtig ist. Denn die Frau war ursprünglich Muslima. Eine Muslima darf nach islamischem Recht keinen Christen heiraten. Auch darf sie nicht zum Christentum übertreten. Die Ehe sei aus diesem Grund unwirksam. Und ein nichteheliches Zusammenleben verstößt gegen die in Ägypten herrschenden Moralvorstellungen.

Vgl. Jan Brugman, De betekenis van het mohammedaanse recht in het hedendaagse Egypte, 's Gravenhage, 1960, S. 19-20.

Hierzu im Überblick z.B. J.N.D. Anderson, Law Reform in Egypt 1850-1950, in: P.J. Vatikiotis, Revolution in the Middle East and Other Case Studies, London, 1972, S. 146-173; Bernard Botiveau, Loi islamique et droit dans les sociétés arabes: Mutations des systèmes juridiques du Moyen-Orient, Paris/ Aix-en-Provence, 1993; Brugman (Anm. 11); Chafik Chehata, Le Droit Moderne de l'Egypte, in: Handbuch der Orientalistik, Ergänzungsbd. 3, Orientalisches Recht, S. 360-386; Farhat J. Ziadeh, Lawyers, the Rule of Law and Liberalism in Modern Egypt, Stanford, 1968.

Urt. vom 30.3.1966, Majmî́'at mahkamat al-naqd 17, S. 782-791. 
Der ägyptische Kassationshof gab dem Vermieter Recht. Was die familienrechtliche Seite des Falles angeht, ist die Rechtslage dabei weitestgehend vorgezeichnet. Wie zuvor erwähnt, richtet sich das Familienrecht in Ägypten weiterhin im Grundsatz nach islamischem Recht. ${ }^{14}$ Das "Ehehindernis der Religionsverschiedenheit", das - unter anderem - einer Muslima verbietet, einen Christen zu heiraten, ist ein allgemeiner Grundsatz des islamischen Familienrechts, der auch heute noch in der ägyptischen Rechtsprechung Anwendung findet. ${ }^{15}$ Dies gilt gleichermaßen für das Verbot des Religionswechsels: einem Muslim ist verwehrt, seinen Glauben aufzugeben. ${ }^{16}$ Was hier aus juristischer Sicht daher weitaus interessanter ist, ist die Frage der Zulässigkeit der Klage: Wie kann es dem Vermieter gestattet sein, auf Feststellung der Nichtigkeit der Ehe seiner Mieter zu klagen? Denn das Prozeßrecht richtet sich auch in familienrechtlichen Streitigkeiten nach der Zivilprozeßordnung (die sich am französischen Vorbild orientiert). Art. 3 der ägyptischen Zivilprozeßordnung (Gesetz Nr. 13/ 1968) bestimmt wiederum: "Eine Klage ist nur dann zulässig ... wenn der Kläger mit ihr ein rechtlich anerkanntes Interesse verfolgt."

Das von der Zivilprozeßordnung geforderte Rechtsschutz- oder Feststellungsinteresse, so der Kassationshof, folge hier jedoch aus dem islamischen Prinzip der hisba: Das allgemeine Rechtsschutzinteresse als Sachurteilsvoraussetzung, so das Gericht, sei eine Frage des Prozeßrechts. Insoweit finde die Zivilprozeßordnung Anwendung. Ob hingegen ein "Interesse" vorliege, sei eine Frage, die mit dem klagweise geltend gemachten Anspruch zusammenhinge. Dies sei daher eine Frage des materiellen Rechts, die - bei einer familienrechtlichen Streitigkeit - nach islamischem Recht zu beurteilen sei. Das Prinzip der hisba sei ein anerkannter Grundsatz des islamischen Rechts. Das islamische Recht gestatte einem jeden Muslim Klage zu erheben, wenn die "Rechte Gottes" verletzt würden. Zu diesen im Interesse der Allgemeinheit bestehenden Vorschriften, so das Gericht weiter, gehöre insbesondere auch das Ehehindernis der Religionsverschiedenheit. ${ }^{17}$ unterliegen familienrechtliche Streitigkeiten unter Muslimen dem islamischen Recht der hanafitischen Schule, soweit diese nicht spezialgesetzlich geregelt sind.

15 Z.B. Kassationshof, Urt. v. 24.12.1975, in: M. 'Abd al-Tawwâb, Al-mustahdath fî qadâ' al-ahwâl al-shakhsiyya, Alexandria, 1991, S. 50-51; vgl. a. Muhammad 'Azmî al-Bakrî, Mawsû'at al-fiqh wa'l-qadâ' fí'l-ahwâl al-shakhsiyya, Kairo, 1994, Bd. 1, S. 232-233 (Rn. 124).

Z.B. Kassationshof, Urt. vom 29.5.1968, Majmû'at Mahkamat al-Naqd 19, 1034-1037; Oberstes Verwaltungsgericht, Urt. vom 25.1.1971, E. 599/ 19, in: Na'îm 'Attiy'ya / Hasan al-Fakahânî, Almawsû'a al-idâriyya al-hadîtha, Kairo, 1986-1987, Bd. 13, S. 395-397; Appellations gericht Kairo, Urt. vom 7.2.1992, in: Dawoud Sudqi El Alami, The Marriage Contract in Islamic Law - in the Shariah and the Personal Status Laws of Egypt and Morocco, London et al., 1992, S. 45-46.

Kassationshof, Urt. vom 29.5.1968 (Anm. 16), S. 788-789. 


\section{III.}

Bei dieser von dem ägyptischen Kassationshof entwickelten "hisba-Rechtsprechung" handelte es sich um einen anerkannten, ${ }^{18}$ wenn auch nicht vollkommen unangefochtenen Grundsatz des ägyptischen Zivilprozeßrechts. ${ }^{19}$ Bis Anfang der 90er Jahre hatte dieses Institut jedoch keine nennenswerte praktische Bedeutung. In das Blickfeld der Öffentlichkeit geriet die hisba erst, als diese Popularklage als Instrument in der politischen Auseinandersetzung zwischen der islamistischen Opposition und dem Staat entdeckt wurde, eine Auseinandersetzung, die gerade in den letzten Jahren vermehrt mit juristischen Mitteln geführt wurde. Diese Entwicklung ist insbesondere mit zwei Fällen verbunden: Dem bereits genannten Fall Abû Zayd und der Auseinandersetzung um den Film "al-Muhâjir" [der Emigrant] des Regisseurs Youssef Chahine.

Der Fall Abû Zayd hatte dabei den folgenden Hintergrund: ${ }^{20}$ Bekannt geworden ist der ägyptische Koranwissenschaftler mit seinem 1990 erschienenen Buch "Mafhûm al-Nass" [Der Begriff Text]. Hierbei handelt es sich um einen Versuch, die Methoden der modernen Sprachwissenschaft für die Interpretation des Korans fruchtbar zu machen. Die zentrale These des (für ein Nichtfachpublikum nur schwer zugänglichen) Buches ist, daß auch koranische Interpretation vom jeweiligen historischen und sozialen Kontext geprägt ist. Der Autor beließ es aber nicht bei wissenschaftlichen Thesen. Vielmehr leitete er aus seinem Plädoyer für eine "dynamische", den heutigen Verhältnissen entsprechende Koraninterpretation auch pointierte politische Aussagen ab und kritisierte insbesondere das von Orthodoxie und Islamisten gleichermaßen beanspruchte "Auslegungsmonopol" in religiösen Fragen. Seine Thesen entfachten in der ägyptischen Öffentlichkeit eine an Polemik schwer zu überbietende Auseinandersetzung, in deren Verlauf von seinen Gegnern der Vorwurf der

Vgl. z.B. Mustafâ Majdî, Al-mawsû'a al-qadâ'iyya fî'l-murâfa'ât al-madaniyya wa'l-tijâriyya, Alexandria, 1995, Bd. 1, S. 53; Anwar Tulba, Mawsû'at al-murâfa'ât al-madaniyya wa'l-tijâriyya, Alexandria, 1993, Bd. 1, S. 50.

Im Gegensatz zum Kassationshof, der in der hisba ein Institut des materiellen Rechts sieht, das daher auch von der Verweisungsvorschrift Art. 280 Gesetz Nr. 78/ 1931 i.V.m. Art. 6 Abs. 1 Gesetz Nr. 462/ 1955 umfaßt wird, stellt die Gegenansicht darauf ab, hierbei handele es sich um ein Institut des Prozeßrechts. In der Zivilprozeßordnung (Gesetz Nr. 13/ 1968) sei dieses Institut im Gegensatz zur früheren Zivilprozeßordnung - nicht geregelt. Der Gesetzgeber habe daher mit der neuen Zivilprozeßordnung die hisba-Klage abschaffen wollen. Zum Meinungsstand ausführlich z.B. Muhammad Shitâ' Abû Sa'd, Al-daf' bi-'adam al-qabûl, Alexandria, 1997, S. 237-250.

$\mathrm{Zu}$ den Hintergründen des Falls ausführlich z.B. Annette Heilmann, Die Affäre Abu Zayd und der Begriff der "Ethik der Toleranz" in der heutigen politischen Diskussion in Ägypten, in: Ferhad Ibrahim (Hrsg.), Staat und Zivilgesellschaft in Ägypten, Hamburg, 1995, S. 145-168; Navid Kermani, Die Affäre Abû Zayd: Eine Kritik am religiösen Diskurs und ihre Folgen, Orient 35 (1994), S. 25-49; Nasr Abu Zaid, "The Case of Abu Zaid", Index on Censorship, Nr. 4/ 1996, S. 30-39. $\mathrm{Zu}$ den vom Fall aufgeworfenen juristischen Fragen ausführlich vgl. die Nachweise oben (Anm. 2). 
Häresie erhoben wurde. Daß Koranauslegung mit modernen Methoden auf erbitterten Widerstand stoßen kann, hat sich in Ägypten auch in der Vergangenheit schon mehrfach gezeigt; der Kairener Literaturwissenschaftler war nicht der erste, dessen modernistische Koraninterpretation heftig angefeindet wurde. ${ }^{21}$ Das besondere am Fall Abû Zayd war nun: Unter Berufung auf das Prinzip der hisba erhob eine Gruppe islamistischer Rechtsanwälte Klage beim Familiengericht. Wie bereits im zuvor geschilderten Fall des Kassationshofs aus dem Jahr 1966 hatte sich dieses bei der Klage auf Feststellung der Nichtigkeit der Ehe inzident mit Fragen der Apostasie auseinanderzusetzen, diesmal anhand der wissenschaftlichen Veröffentlichungen Abû Zayds. Hierbei handelte es sich auch um das eigentliche Ziel der Kläger, wie diese gegenüber der Presse freimütig einräumten. ${ }^{22}$

Der Fall Abû Zayd war dabei nicht der einzige Fall seiner Art. ${ }^{23}$ Auch Youssef Chahine, Ägyptens bekanntester Regisseur und 1997 bei dem Festival von Cannes für sein Lebenswerk ausgezeichnet, sah sich mit einer ähnlich gelagerten gerichtlichen Auseinandersetzung konfrontiert. ${ }^{24}$ Hier begehrte der Kläger ein Verbot des Films "al-Muhâjir", der im Herbst 1994 mit großem Erfolg in den ägyptischen Kinos angelaufen war. Zur Begründung trug er vor, der Protagonist des dreistündigen Epos aus der Pharaonenzeit porträtiere den Propheten Joseph. Dies verstoße gegen das islamische Bilderverbot. Der Film verletze daher die religiösen Gefühle der Ägypter. Das Gericht gab der Klage statt, und der Film durfte in ägyptischen Kinos nicht mehr gezeigt werden. ${ }^{25}$ Auch in diesem Fall wurde das Rechtsschutzinteresse des Klägers mit dem Prinzip der hisba begründet: "Die Klage betrifft einen Propheten Gottes, von daher ist sie als hisba-Klage zulässig." ${ }^{26}$ Dies, obgleich in Ägypten Filme in moralischer, politischer und religiöser Hinsicht auch staatlicherseits einer umfassenden Kontrolle unterliegen. ${ }^{27}$

Dies zeigen die Auseinandersetzungen um die Werke von Taha Husayn, Amîn al-Khûlî, Muhammad Ahmad Khalafallâh und, in den letzten Jahren, Muhammad Sa'îd al-'Ashmâwî und Hassan Hanafî (hierzu vgl. Donald Malcolm Reid, Cairo University and the Making of Modem Egypt, Cambridge et al., 1990, S. 120-125, 154 f., Bernard Botiveau, Penser, dire et interdire: logiques et en jeux de la censure des écrits en Egypte, Egypte / monde arabe, Nr. 14 (1993), S. 133-162). Vgl. Middle East Times Egypt, 6.-12.7.1993.

Von ähnlichen Verfahren war eine ganze Reihe von ägyptischen Intellektuellen betroffen, u.a. auch der Literaturnobelpreisträger Nagib Mahfuz. Diese Verfahren haben sich jedoch sämtlich aufgrund einer Gesetzesänderung im Januar 1996 erledigt (zum neuen hisba-Gesetz ausführlich unter IV.).

Hierzu ausführlich Nathalie Bernard-Maugiron, Anatomie sociologique d'une affaire égyptienne: le procés de "l Emigré", de Youssef Chahine, in: Boëtsch / Dupret / Ferrié (Anm. 2), S. 162-192. Inzwischen ist das Verbot Zeitungsberichten zufolge jedoch aufgehoben worden (vgl. Al-Ahram Hebdo, 6.-12. August 1997). 
IV.

Die Beispiele aus der neueren ägyptischen Rechtsprechung zeigen, daß das prozessuale Institut der hisba ausgesprochen mißbrauchsanfällig ist. Ob angeblich häretische Koraninterpretation oder ein vermeintlicher Verstoß gegen das islamische Bilderverbot: Hier zeichnet sich eine Tendenz ab, daß Gerichte für politische Zwecke in Anspruch genommen werden, indem die Rechtgläubigkeit einzelner einer gerichtlichen Nachprüfung unterzogen wird.

Vom ägyptischen Parlament wurde daher im Januar 1996 in aller Eile ein neues Gesetz zur "Regelung des Verfahrens der hisba-Klage in Personalstatutsangelegenheiten" 28 erlassen. Der Zweck dieses Gesetzes, so die Stellungnahme des Parlaments, bestand darin, "geistigen Terrorismus [al-irhâb al-fikrî] zu bekämpfen." ${ }^{29}$ Hierdurch sollten, "Extremisten, die für sich in Anspruch nehmen, die einzigen rechtgläubigen Muslime zu sein" davon abgehalten werden, "Intellektuelle zu beschädigen." 30

Im einzelnen enthält das Gesetz die folgenden Regelungen:

1. Die Befugnis zur Erhebung der hisba-Klage wird auf die niyâba, die Staatsanwaltschaft, übertragen. Dieser sind vermeintliche Verstöße gegen die "Rechte Gottes" anzuzeigen. Die Staatsanwaltschaft ermittelt den Sachverhalt von Amts wegen und entscheidet dann über die Klageerhebung (Artt. 1, 2).

2. Wird eine hisba-Klage erhoben, tritt die Staatsanwaltschaft im Prozeß als Kläger auf. Derjenige, der die Anzeige erstattet hat, ist nicht Beteiligter des Verfahrens (Artt. 3, 4).

Darüber hinaus wurde der Art. 3 der Zivilprozeßordnung folgendermaßen geändert. ${ }^{31}$

1. Eine Klage ist nur dann zulässig, wenn der Kläger mit ihr ein "eigenes und unmittelbares" Interesse verfolgt (Art. 3 Abs. 1).

2. Im Falle einer mißbräuchlichen Klageerhebung kann ein Ordnungsgeld in Höhe von bis zu 500 ägyptischen Pfund ${ }^{32}$ verhängt werden (Art. 3 Abs. 4).

Damit wurde die hisba als eine Art der Popularklage, die dem "engagierten Bürger und Gläubigen" ermöglicht "unislamisches Verhalten" vor Gericht zu bringen, abgeschafft. Die

Gesetz Nr. 3/ 1996, Al-jarîda al-rasmiy'ya [Gesetzesblatt] Nr. 4 vom 29. Januar 1996.

Al-Ahrâm, 30.1.1996.

A.a.O.

Gesetz Nr. 81/ 1996, Al-jarîda al-rasmiyy'a Nr. 19 vom 22. Mai 1996.

Ungefähr 250,- DM. 
Befugnis der hisba steht fortan ausschließlich der Staatsanwaltschaft zu. Diese wird zum modernen muhtasib. Damit wird jedoch nicht nur eine Institution des modernen Territorialstaates durch die Rückführung auf eine Institution des traditionellen islamischen Rechts mit einer islamischen Legitimation versehen. ${ }^{33}$ Vielmehr wird auch die Definition der durch die hisba zu wahrenden islamischen Ordnung einer unmittelbaren staatlichen Kontrolle unterworfen. Denn welche Verstöße gegen die islamische Ordnung Anlaß zum Einschreiten gebieten, bestimmt fortan die Staatsanwaltschaft.

V.

Bei der Auseinandersetzung um die hisba geht es daher um mehr als nur um Feinheiten des ägyptischen Zivilprozeßrechts. Im Kern geht es hier vielmehr um die Frage, wer im modernen Territorialstaat die Kompetenz besitzt zu definieren, was unter "Islam" und "islamischer Ordnung" zu verstehen ist. Daß diese Frage dabei älter ist als der moderne Territorialstaat, hat der historische Rückblick zu Anfang gezeigt: Bereits der frühere muhtasib Ibn alUkhuwwa hat sich mit dem Problem beschäftigt, wer denn nun eigentlich dazu berufen ist, das "Gute zu befördern und das Schlechte zu verhindern." Die bis heute fortbestehende Aktualität dieser Frage zeigen die Beispiele aus der jüngeren ägyptischen Rechtsprechung.

Mit dem ägyptischen hisba-Gesetz von 1996 ist hierauf nun - zumindest vordergründig eine eindeutige Antwort gefunden worden: Was unter "Islam" und "islamischer Ordnung" zu verstehen ist, wird von den Institutionen des modernen Territorialstaates verbindlich festgelegt. Nicht der einzelne und auch nicht die islamischen Juristen, herkömmlicherweise mit der Auslegung des islamischen Rechts betraut, sondern der Staat ist zu dessen autoritativer Auslegung berufen.

Dies ist eine Tendenz, die sich im übrigen nicht auf die Frage der hisba beschränkt. Auch auf verfassungsrechtlichem Gebiet läßt sich in Ägypten eine ähnliche Entwicklung konstatieren. Art. 2 der ägyptischen Verfassung von 1971 (i.d.F. v. 1980) bestimmt: "Die Grundsätze des islamischen Rechts sind die Hauptquelle der Gesetzgebung." Ausgehend von diesem Verfassungsgrundsatz hat der ägyptische Verfassungsgerichtshof seit Mitte der 80er Jahre eine differenzierte Rechtsprechung entwickelt, unter welchen Voraussetzungen vom Staat erlassene Gesetze verfassungswidrig sind, weil sie gegen die "Grundsätze des islami-

Diese Tendenz zeigt sich hingegen z.B. in dem marokkanischen "Loi no. 02-82 relative aux attributions du muhtasib et des umanâ' des corporations", Dahir no. 1-82-70 vom 21. Juni 1982 (hierzu ausführlich Michèle Zirari-Devif, La hisba au Maroc: hier et aujourd'hui, in: Hervé Bleuchot (ed.), Les institutions traditionelles dans le monde arabe, Paris / Aix-en-Provence, 1996, S. 71-85). 
schen Rechts verstoßen." ${ }^{34}$ In diesem Zusammenhang nimmt das oberste ägyptische Gericht für sich in Anspruch, selbst die einschlägigen Bestimmungen von Koran und Sunna auszulegen und deren autoritative Lesart verbindlich festzuschreiben.

Diesem Aspekt der Kontrolle religiöser Tradition durch die Institutionen des modernen Territorialstaats ist gerade bei der Bewertung des neuen hisba-Gesetzes bislang viel zu wenig Rechnung getragen worden. Bassam Tibi, zum Beispiel, bezeichnete dieses als "Gesetz zur Bekämpfung des Unglaubens," das die Handhabe biete, "die Scharia einzuführen" ${ }^{35}$. Dabei geht Tibi offensichtlich davon aus, hier würden die Institutionen des modernen Territorialstaats im Zuge einer "Reislamisierung" grundsätzlich in Frage gestellt. Das Gegenteil ist der Fall: Denn das hisba-Gesetz ist ein Instrument des Staates, eine einheitliche Vorstellung von islamischer Ordnung verbindlich vorzuschreiben. Also keine "Islamisierung der ägyptischen Rechtsordnung," wie dies oft zu lesen ist, ${ }^{36}$ sondern gewissermaßen eine "Verstaatlichung des islamischen Rechts."

Hierzu ausführlich Baudouin Dupret, A propos de la constitutionnalité de la sharî'a. Présentation et traduction de l'arrêt de 26 mars 1994 (14 Shawwâl 1414) de la Haute Cour Constitutionelle (almahkama al-dustûriyya al-'ulyâ) égyptienne, Islamic Law and Society, 4 (1997), S. 91-113; Kilian Bälz. Islamisches Recht, staatliche Rechtsetzung und verfassungsgerichtliche Kontrolle. Der ägyptische Verfassungsgerichtshof und der Schleier in staatlichen Schulen (Urt. 8/ 17 vom 18.5.1996), Zeitschrift für ausländisches öffentliches Recht und Völkerrecht 57 (1997), S. 229242, jeweils mit Nachweisen der Rechtsprechung.

"Demokratie ist Unglaube. In Ägypten bietet ein Gesetz zur Bekämpfung des Unglaubens die Handhabe, die ,Scharia“" einzuführen, Frankfurter Allgemeine Zeitung vom 3. Juli 1996. Rechtssystems, Süddeutsche Zeitung, 8. Februar 1996 (die dortige Darstellung der Diskussion ist jedoch differenzierter als der Titel nahelegt). 
vival by covering themselves of the support of important interest groups and at the same time of deterring others from taking over power.

Personalization of political decisions and politicization of economy and administration as well as personalization and privatization of the juridical sector are necessary political elements to maintain power in authoritarian regimes leading all to and requiring widespread juridical insecurity. As economic and political development can hardly be expected in such authoritarian regimes, the introduction of more democratic and constitution based elements and the implementation of market economic structures are necessary.

\title{
Constitutional Jurisdiction in Guatemala
}

\author{
By Francisco Fernández Segado
}

In Guatemala, the peace treaty signed on December 29, 1996 by President Alvaro Arzú, heading the peace-commission COPAZ, and by the leaders of the URNG, merging four major guerilla-groups, ended a six-year peace marathon which followed 36 years of armed conflict, the last contemporary armed conflict to be solved in Central America.

Nevertheless, 12 years ago, on July 1, 1984, general, fair and free elections for a constitutional assembly took place. This constituante managed to draw up a new constitution within 10 months, which was then promulgated on May 31, 1985. The paper focuses on the newly created constitutional court of Guatemala and, in comparison with the preceding constitution of 1966, on the legal framework attributing this court the powers to protect both human rights and the constitutionality of the political process in general.

The author gives an extensive overview which may serve as a solid base for the upcoming discussion, whether or not democratically legitimated constitutional jurisprudence is able to play an important role in enforcing the peace treaty implementation process within the country.

\section{Popular Action in defense of "Divine Law": Hisba in contemporary Egypt}

\section{By Kilian Bälz}

The paper investigates the Islamic principle of hisba in contemporary Egypt. In examining recent Egyptian case-law, the paper argues that the traditional concept of hisba, the Islamic duty to "promote good and prevent evil", underwent a fundamental transformation: traditionally, the duty of hisba rested upon the public function of the muhtasib ["market supervisor"]. Today, in contrast, the principle is employed to entitle a third party to an action in 
cases related to religion and public morality. In early 1996, however, the Egyptian government enacted a new "Law Regulating the hisba-Action". Through this legislative enactment the individual's right to raise an action based on hisba was abolished and the public attorney was installed as modern muhtasib. This law, therefore, conforms to the general tendency of submitting the definition of religious traditions under the control of the nation state in contemporary Egypt. 